\title{
Lagartos (Squamata: Lacertilia) em áreas de floresta e de pastagem em Espigão do Oeste, Rondônia, sudoeste da Amazônia, Brasil
}

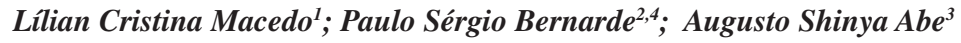 \\ ${ }^{1}$ Laboratório de Ecologia Animal, Faculdade de Ciências Biomédicas de Cacoal - FACIMED, \\ Av. Cuiabá 3087, Jardim Clodoaldo, CEP 78976-005, Cacoal, RO, Brasil, \\ http://www.facimed.com.br, e-mail: rhinella@hotmail.com \\ ${ }^{2}$ Laboratório de Herpetologia, Centro Multidisciplinar, Campus Floresta, \\ Universidade Federal do Acre - UFAC, CEP 69980-000, Cruzeiro do Sul, AC, Brasil \\ ${ }^{3}$ Departamento de Zoologia, Instituto de Biociências, Universidade Estadual Paulista - UNESP, \\ CP 199, CEP 13506-900, Rio Claro, SP, Brasil, http://www.rc.unesp.br, e-mail: asabe@rc.unesp.br \\ ${ }^{4}$ Autor para correspondência: Paulo Sérgio Bernarde, e-mail: snakebernarde@hotmail.com
}

Macedo, L. M.; Bernarde, P. S; Abe, A. S. Lizard community (Squamata: Lacertilia) in forest and pasture areas at Espigão do Oeste, Rondônia, southwestern Amazon, Brazil. Biota Neotrop., vol. 8, no. 1, Jan./Mar. 2008. Available at: <http://www.biotaneotropica.org.br/v8n1/en/abstract?inventory+bn01108012008>.

\begin{abstract}
This study describes the richness and capture rates of lizards in forested and pasture areas at a locality in Rondônia (southwestern Amazon) utilizing three sampling methods: pitfall trap with drift fences, nocturnal time-constrained search and incidental finding. In all, 29 species belonging to nine families was recorded. Most species were found in forested area, whilst only eight species in pasture area. Most species were collected with pitfall trap (22), followed by incidental finding (16), and nocturnal search (9). The collecting curve gathering the three methods used showed that after the eighth month of sampling no new species could be added. This data suggests that the community was well sampled. The fact that some species were collect just by one of collecting method indicates the adequacy of using two or more methods for sampling communities. Higher species richness was recorded in forested areas compared with pastures. Such reduction is species number is due to the removing of plant covering and its consequences, the increasing predation rate, competition, food reduction, losses of sheltering and breeding, and limited thermoregulatory capacity.
\end{abstract}

Keywords: Reptilia, Lacertilia, species richness, sampling methods.

Macedo, L. M.; Bernarde, P. S.; Abe, A. S. Lagartos (Squamata: Lacertilia) em áreas de floresta e de pastagem em Espigão do Oeste, Rondônia, sudoeste da Amazônia, Brasil. Biota Neotrop., vol. 8, no. 1 jan./mar. 2008. Disponível em: <http://www.biotaneotropica.org.br/v8n1/pt/abstract?inventory+bn01108012008>.

Resumo: O presente estudo apresenta dados sobre riqueza e freqüência de captura de lagartos em áreas de floresta e pastagem em uma localidade em Rondônia (sudoeste da Amazônia) utilizando três métodos de amostragem: armadilhas de interceptação e queda, procura noturna limitada por tempo (encontro de espécimes dormindo sobre a vegetação) e encontros ocasionais. Foram encontradas 29 espécies distribuídas em nove famílias. A maioria das espécies (28) foi encontrada em floresta, enquanto que na pastagem foram encontradas oito. A maioria das espécies foi registrada pelas armadilhas de interceptação e queda (22), seguido pelos encontros ocasionais (16) e procura noturna (9). A curva do coletor apresentando dados de todos os métodos mostra que a partir do oitavo mês de amostragem não houve mais nenhum acréscimo de nova espécie nessa comunidade, evidenciando que a mesma foi bem amostrada. $\mathrm{O}$ fato de algumas espécies terem sido coletadas exclusivamente em apenas um dos métodos, demonstra a importância de se usar dois ou mais métodos de amostragem em estudos sobre comunidades. Essa redução de espécies se deve a retirada da cobertura vegetal e, possivelmente, às suas conseqüências: aumento das taxa de predação e de competição, dificuldades para a termo-regulação, perda de locais para abrigos e reprodução, diminuição dos recursos alimentares e perda de serapilheira.

Palavras-chaves: Répteis, Lacertilia, riqueza de espécies, métodos de amostragem. 


\section{Introdução}

Atualmente, são conhecidas cerca de 98 espécies de lagartos na Amazônia brasileira, incluindo os enclaves de Cerrado (Ávila-Pires 1995, Ávila-Pires \& Vitt 1998, Ávila-Pires \& Hoogmoed 2000, Gainsbury \& Colli 2003, Rodrigues \& Avila-Pires 2005, Mesquita et al. 2006). No entanto, apesar da riqueza da fauna de lagartos na Amazônia ser a maior entre os biomas brasileiros (Rodrigues 2005), ainda são necessárias mais informações para uma melhor compreensão dessa biodiversidade (Vogt et al. 2001, Rodrigues 2005, Ávila-Pires et al. 2007).

O Estado de Rondônia é considerado uma área de intrincada interação de faunas, fato relacionado com a complexidade de sua história ambiental e geológica (Ávila-Pires 1995). Dentre os estudos realizados com lagartos nesse estado, destacam-se os levantamentos de espécies em localidades ao longo do trecho da BR-364 durante a execução do Projeto Polonoroeste (Vanzolini 1986, Nascimento et al. 1988) e aqueles realizados por Yuki et al. (1999) e Brandão (2002) nos municípios de Pimenta Bueno e Costa Marques, respectivamente. No entanto, nenhuma localidade no estado foi bem amostrada quanto à riqueza específica de lagartos, pois Vanzolini (1986) e Nascimento et al. (1988) coletaram em vários municípios e Yuki et al. (1999) e Brandão (2002) apresentaram resultados de inventários rápidos. Estudos enfocando diferentes aspectos da ecologia de populações de algumas espécies também foram desenvolvidos em Rondônia (Vitt \& Caldwell 1993, Vitt \& Colli 1994, Vitt et al. 2005). As comunidades de lagartos em três áreas de Cerrado dos municípios de Guajará-Mirim, Pimenta Bueno e Vilhena foram estudadas por Gainsbury \& Colli (2003).

Uma das principais ameaças à fauna de lagartos é a destruição dos habitats (Rodrigues 2005), sendo que o Estado de Rondônia foi sugerido como uma área de interesse para estudos sobre a diversidade de répteis devido à intensa atividade antrópica e conseqüente degradação ambiental (Vogt et al. 2001). Na Amazônia, clareiras e trilhas originadas devido à exploração de madeira beneficiaram algumas espécies de lagartos heliotérmicas que puderam predar ou competir com algumas espécies de lagartos florestais (Vitt et al. 1998, Vitt \& Caldwell 2001). Poucos estudos relatando a diferença de riqueza da herpetofauna entre áreas florestadas e de lavouras e/ou pastagens foram desenvolvidos no Brasil (e.g., Bernarde et al. 1999, Bernarde 2007, Gardner et al. 2007). Comparações da herpetofauna de serapilheira entre áreas de floresta (primária e secundária) e de plantações de Eucalyptus spp. no Pará revelaram uma dominância de espécies de lagartos de área aberta nesse último ambiente (Gardner et al. 2007). Em Rondônia, com a transformação de florestas em áreas de pastagens, foi observada uma diminuição no número de espécies de anfíbios (Bernarde et al. 1999, Bernarde 2007) e de serpentes (Bernarde \& Abe 2006) em algumas áreas.

O presente estudo investiga a riqueza e a frequiência de captura de lagartos em uma localidade no município de Espigão do Oeste, Estado de Rondônia (sudoeste da Amazônia), comparando áreas de floresta e de pastagem.

\section{Material e Métodos}

Área de estudo: $\mathrm{O}$ estudo foi desenvolvido na Fazenda Jaburi $\left(11^{\circ} 35^{\prime}-11^{\circ} 38^{\prime} \mathrm{S}\right.$ and $\left.60^{\circ} 41^{\prime}-60^{\circ} 45^{\prime} \mathrm{W}\right)$, localizada no quilômetro 32 da Rodovia do Calcário, no município de Espigão do Oeste, Estado de Rondônia, Brasil (Figura 1). Esta propriedade rural apresenta uma área de 4000 hectares, dos quais 50\% correspondem à reserva legal (Bernarde \& Abe 2006, Bernarde 2007). A altitude da região varia em torno de $280 \mathrm{~m}$ acima do nível do mar. A região apresenta média pluviométrica anual de $2300 \mathrm{~mm}$, com um período de seca geralmente entre abril e setembro, e temperatura média anual de $26^{\circ} \mathrm{C}$ (os dados climáticos foram obtidos através da Prefeitura Municipal de Espigão do Oeste). $\mathrm{O}$ ano pode ser dividido em um período com meses mais

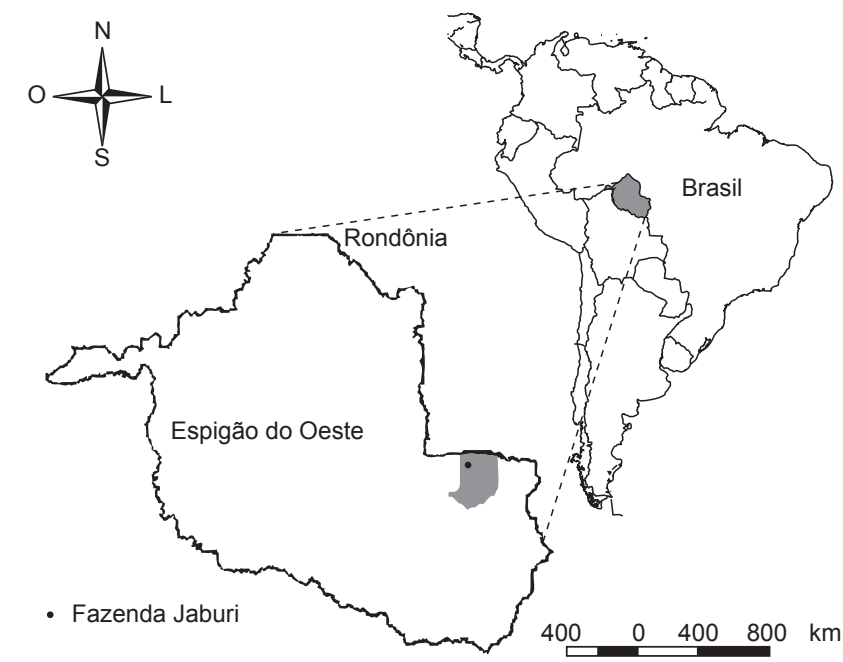

Figura 1. Mapa da América do Sul mostrando a localização do Município de Espigão do Oeste, Estado de Rondônia, Brasil.

Figure 1. Map of South America showing the localization of Municipality of Espigão do Oeste in Rondônia State, Brazil.

chuvosos (outubro a março $=1850 \mathrm{~mm}$ ) e outro com meses mais secos (abril a setembro $=456 \mathrm{~mm}$ ). A vegetação se enquadra no Domínio Equatorial Amazônico “Amazônia” (sensu Ab’Saber 1977), com vegetação do tipo Floresta Ombrófila Aberta Submontana (Oliveira 2002). Nas áreas de pastagem existe o predomínio da gramínea Brachiaria brizantha, com poucos arbustos e árvores.

Coleta dos dados: A fase de preparação para a coleta de dados (reconhecimento dos ambientes, instalação das armadilhas e abertura de trilhas) ocorreu nos meses de fevereiro e março de 2001. As amostragens foram mensais entre abril de 2001 e março de 2002. Foram empregados três métodos:

1) Procura noturna limitada por tempo (Campbell \& Christman 1982): Consistiu no deslocamento a pé, lentamente, através de trilhas (dentro de mata) e estradas (área de pastagem), à procura de lagartos que estivessem visualmente expostos. O esforço de procura abrangeu todos os microhábitats visualmente acessíveis (chão, troncos caídos, vegetação). Em cada noite, foram percorridos trechos de 300 a 400 m durante um período de três a quatro horas, respectivamente (entre 18:00 e 23:00 horas), durante oito a dez noites por mês. Na floresta a procura foi realizada em duas trilhas (400 e $1200 \mathrm{~m}$ ) e na pastagem em estradas. Foram realizadas 384 horas-homem de procura dentro de matas e 384 horas-homem em áreas de pastagem, totalizando 768 horas-homem durante um ano (64 horas por mês).

2) Armadilhas de interceptação e queda com cerca-guia (Campbell \& Christman 1982, Cechin \& Martins 2000): Neste método foram utilizados tambores plásticos (200 litros, com $90 \mathrm{~cm}$ de altura), enterrados a cada dez m e interceptados por uma cerca-guia de lona plástica com um metro de altura. Foram instaladas seis séries de armadilhas em linha reta. Cada série consistiu de quatro tambores, com uma cerca-guia de $44 \mathrm{~m}$, totalizando 24 tambores. Nos tambores foram feitos pequenos orifícios no fundo para evitar o armazenamento de água das chuvas, prevenindo que os animais capturados se afogassem. Além disso, foi colocado dentro de cada tambor um pedaço de isopor para que os animais pudessem permanecer sobre ele, caso armazenasse água.

As armadilhas de interceptação e queda foram instaladas em três tipos de ambiente (distância mínima de 1800 m entre eles), com duas séries em cada: dentro de floresta, distante de corpos d'água permanentes; dentro de floresta, a cerca de $100 \mathrm{~m}$ de 
um rio (cerca de $4 \mathrm{~m}$ de largura e $0,80 \mathrm{~m}$ de profundidade) e em área de pastagem, distante de corpos d'água permanentes. Em cada ambiente, as séries de armadilhas foram instaladas à distância de aproximadamente $200 \mathrm{~m}$ entre si. As armadilhas permaneceram abertas ininterruptamente durante um ano (730/ horas/balde/mês) e foram monitoradas no máximo a cada dois dias pela manhã.

3) Encontros ocasionais: Foram considerados os espécimes de lagartos encontrados durante a realização de outras atividades, como por exemplo, no deslocamento para vistoriar as armadilhas. Cerca de 50 horas mensais foram despendidas nessa atividade, totalizando 600 horas durante um ano. Esse método não foi utilizado para a estimativa da frequiência da espécie, mas apenas para o registro de sua ocorrência nos ambientes avaliados.

Para a identificação dos espécimes utilizou-se a chave taxonômica e as descrições de Ávila-Pires (1995). Alguns espécimes foram coletados como material testemunho e foram depositados na Coleção Herpetológica do Museu Paraense Emílio Goeldi (MPEG) (ver relação em Apêndice).

A curva do coletor, geral e para cada método separadamente, foi calculada para avaliar se a taxocenose dessa localidade foi bem amostrada.

\section{Resultados}

Foram encontradas 29 espécies de lagartos distribuídas em nove famílias : Hoplocercidae (1 espécie), Iguanidae (1), Polychrotidae (5), Leiosauridae (1), Tropiduridae (5), Gekkonidae (5), Gymnophthalmidae (6), Teiidae (4) e Scincidae (1) (Tabela 1). A maioria das espécies (28) foi encontrada em floresta, enquanto na pastagem foram registradas apenas oito (Tabela 1).

A maioria das espécies foi registrada pelas armadilhas de interceptação e queda (22), seguido pelos encontros ocasionais (16) e procura noturna limitada por tempo (9) (Tabela 1). Oito espécies foram obtidas exclusivamente pelas armadilhas de interceptação e queda, sete pelos encontros ocasionais e nenhuma foi registrada somente pela procura noturna (Tabela 1). O único método que atingiu a assíntota na curva do coletor foi o de encontros ocasionais (Figura 2). Contudo, a curva do coletor apresentando dados de todos os métodos mostra que a partir do oitavo mês de amostragem não houve acréscimo de nova espécie nessa comunidade (Figura 2).

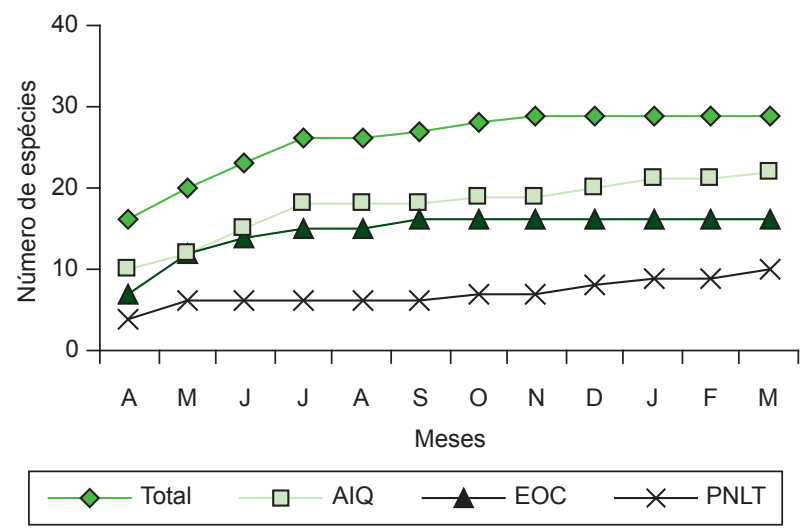

Figura 2. Curva do coletor dos métodos de amostragem utilizados entre o período de abril de 2001 a março de 2002 em Espigão do Oeste, Rondônia, Brasil. Total = todos os métodos; $\mathrm{AIQ}=$ armadilhas de interceptação e queda; $\mathrm{PNLT}=$ procura noturna limitada por tempo $\mathrm{EOC}=$ encontros ocasionais.

Figure 2. Collector curve of the sampling methods used between April of 2001 to March of 2002 period at Espigão do Oeste, Rondônia, Brazil. Total $=$ all methods; AIQ = Pitfall traps with drift fences; PNLT = Nocturnal time-constrained search; EOC = Incidental finding.
As armadilhas de interceptação e queda registraram 18 espécies (108 espécimes) dentro da floresta distante de corpos d'água, 14 (62) na floresta com rio próximo e 5 (67) na área de pastagem (Tabela 2). As cinco espécies de lagartos mais capturadas nas armadilhas foram Ameiva ameiva (58 espécimes), Kentropyx pelviceps (33), Gonatodes hasemani (29), Cercosaura ocellata bassleri (19) e Mabuya nigropunctata (17) (Tabela 2). A freqüência de captura das espécies foi diferente em cada um dos ambientes amostrados (Tabela 2), sendo as seguintes espécies mais freqüentes: K. pelviceps (floresta), G. hasemani (floresta com rio) e A. ameiva (pastagem).

Dentre as espécies registradas pela procura noturna, as cinco mais freqüentes foram Anolis fuscoauratus (19 espécimes), seguida de A. punctatus (9), A. nitens tandai (6), Gonatodes humeralis (5) e A. transversalis (4) (Tabela 1).

\section{Discussão}

O levantamento da fauna de lagartos em Espigão do Oeste (RO) revelou algumas novas ocorrências entre as 29 espécies registradas, uma das maiores riquezas relatadas para a Amazônia (veja Duellman 1990, Vogt et al. 2001, Gardner et al. 2007). Considerando que a curva do coletor incluindo todos os métodos de amostragem utilizados se estabilizou, é possível concluir que essa localidade foi bem amostrada. As espécies Anolis nitens tandai e Stenocercus roseiventris constituem novas ocorrências para o Estado. A primeira era conhecida para os estados do Amazonas (Ávila-Pires 1995), Acre e Pará (Santos-Jr et al. 2007), e a segunda para o Acre, Peru, Bolívia e Argentina (Ávila-Pires 1995). Oito espécies de Stenocercus são conhecidas para o Brasil, três para a Amazônia e outras cinco para o cerrado (Nogueira \& Rodrigues 2006). Stenocercus sinesaccus ocorre nos cerrados do Brasil Central e também foi registrada para Pimenta Bueno e Vilhena, em Rondônia (Gainsbury \& Colli 2003, Nogueira \& Rodrigues 2006). Em Espigão do Oeste essa espécie foi registrada dentro de floresta, a cerca de $90 \mathrm{~km}$ da localidade de cerrado de Pimenta Bueno estudada por Gainsbury \& Colli (2003). Vanzolini (1986) não especificou qual subespécie de Cercosaura ocellata foi coletada em Rondônia (Ávila-Pires 1995), mas a ocorrência de Cercosaura o. bassleri foi confirmada para o estado.

Algumas espécies foram registradas exclusivamente por um dos métodos empregados, demonstrando a importância da utilização de mais de um método de amostragem em inventários desse tipo (Cechin \& Martins 2000, Gardner et al. 2007). As armadilhas de interceptação e queda tendem a amostrar mais espécies de lagartos terrícolas do que arborícolas (Cechin \& Martins 2000, Gardner et al. 2007). O presente estudo corroborou essa tendência com a captura de $82 \%$ de espécimes terrícolas. Entretanto, apenas $61 \%$ das espécies registradas nas armadilhas apresentaram esse hábito. Isso demonstra que esse método também é importante para o registro de espécies arborícolas ou subarborícolas que também freqüentam o solo das florestas. Apesar de Anolis nitens tandai ser uma espécie observada freqüentemente na serapilheira (Vitt et al. 2001), no presente estudo apenas um espécime foi capturado nas armadilhas de interceptação e queda, enquanto seis o foram por procura noturna. Todos os espécimes de Kentropyx pelviceps foram capturados na floresta distante de corpos d'água, o que sugere que esta espécie não esteja associada a rios, conforme já observado em outros estudos (Veja Ávila-Pires 1995). Stenocercus roseiventris é uma espécie associada a ambientes com rochas e próximos de rios (Ávila-Pires 1995), e no presente estudo foi mais capturada em armadilhas na floresta próxima ao rio, que também apresenta rochas. Na pastagem, a espécie Ameiva ameiva, heliotérmica e associada a áreas abertas (Ávila-Pires 1995, Sartorius et al. 1999) foi a mais amostrada. 
Tabela 1. Espécies de lagartos registradas de acordo com o método de amostragem e a ocorrência em áreas de florestas e de pastagens em Espigão do Oeste, Rondônia, Brasil. AIQ = Armadilhas de interceptação e queda; PNLT = Procura noturna limitada por tempo; EOC = Encontros ocasionais; FLO = Florestas; PAST $=$ Pastagens. $+=$ ocorrência; $\mathrm{NQ}=$ não quantificado.

Table 1. Lizards species registered according to the sampling method and occurrence in forests and pastures áreas at Espigão do Oeste, Rondônia, Brazil. $\mathrm{AIQ}=$ Pitfall traps with drift fences; $\mathrm{PNLT}=$ Nocturnal time-constrained search $; \mathrm{EAC}=$ Incidental finding; FLO = Forests; PAST = Pastures. $+=$ occurrence; $\mathrm{NQ}=$ no quantified.

\begin{tabular}{|c|c|c|c|c|c|}
\hline Espécie/ Método & AIQ & PNLT & EOC & FLO & PAST \\
\hline \multicolumn{6}{|l|}{ HOPLOCERCIDAE } \\
\hline Enyalioides laticeps (Guichenot, 1855) & 1 & 1 & - & + & - \\
\hline \multicolumn{6}{|l|}{ IGUANIDAE } \\
\hline Iguana iguana (Linnaeus, 1758) & - & - & + & + & - \\
\hline \multicolumn{6}{|l|}{ POLYCHROTIDAE } \\
\hline Anolis fuscoauratus Duméril \& Bibron, 1837 & 7 & 19 & + & + & - \\
\hline Anolis nitens tandai Ávila-Pires, 1995 & 1 & 6 & + & + & - \\
\hline Anolis ortonii Cope, 1869 & - & - & + & + & - \\
\hline Anolis punctatus Daudin, 1802 & 7 & 9 & - & + & - \\
\hline Anolis transversalis Duméril, 1851 & 1 & 4 & - & + & - \\
\hline \multicolumn{6}{|l|}{ LEIOSAURIDAE } \\
\hline Enyalius leechii (Boulenger, 1885) & 1 & 2 & - & + & - \\
\hline \multicolumn{6}{|l|}{ Tropiduridae } \\
\hline Plica plica (Linnaeus, 1758) & 3 & 1 & - & + & - \\
\hline Plica umbra (Linnaeus, 1758) & 2 & - & - & + & - \\
\hline Stenocercus roseiventris Duméril \& Bibron, 1837 & 6 & - & - & + & - \\
\hline Stenocercus sinesaccus Torres-Carvajal, 2005 & - & - & + & + & - \\
\hline Uranoscodon superciliosus (Linnaeus, 1758) & - & - & + & + & - \\
\hline \multicolumn{6}{|l|}{ GEKKONIDAE } \\
\hline Coleodactylus amazonicus (Anderson, 1918) & 9 & - & + & + & - \\
\hline Gonatodes hasemani Griffin, 1917 & 29 & - & + & + & + \\
\hline Gonatodes humeralis (Guichenot, 1855) & 7 & 5 & + & + & + \\
\hline Hemidactylus mabouia (Moreau de Jonnès, 1818) & - & - & + & - & + \\
\hline Thecadactylus rapicauda (Houttuyn, 1782) & - & - & + & + & + \\
\hline \multicolumn{6}{|l|}{ GYMNOPHTHALMIDAE } \\
\hline Alopoglossus angulatus (Linnaeus, 1758) & - & - & + & + & - \\
\hline Cercosaura argulus Peters, 1863 & 8 & - & - & + & - \\
\hline Cercosaura eigenmanni (Griffin, 1917) & 8 & - & + & + & + \\
\hline Cercosaura ocellata bassleri Ruibal, 1952 & 19 & - & - & + & - \\
\hline Iphisa elegans Gray, 1851 & 10 & - & - & + & - \\
\hline Leposoma osvaldoi Ávila-Pires, 1995 & 4 & - & + & + & - \\
\hline \multicolumn{6}{|l|}{ TEIIDAE } \\
\hline Ameiva ameiva (Linnaeus, 1758) & 58 & - & + & + & + \\
\hline Kentropyx calcarata Spix, 1825 & 4 & - & - & + & - \\
\hline Kentropyx pelviceps Cope, 1868 & 33 & - & - & + & - \\
\hline Tupinambis teguixin (Linnaeus, 1758) & 2 & - & - & + & + \\
\hline \multicolumn{6}{|l|}{ SCINCIDAE } \\
\hline Mabuya nigropunctata (Spix, 1825) & 17 & - & + & + & + \\
\hline Total de espécimes & 237 & 47 & NQ & NQ & NQ \\
\hline Total de espécies & 22 & 8 & 16 & 28 & 8 \\
\hline Riqueza amostrada (\%) & 75,8 & 27,6 & 55,2 & 96,5 & 27,5 \\
\hline
\end{tabular}

As maiores riquezas foram registradas nos ambientes florestais, $o$ que sugere que ocorra perda de espécies nos ambientes de pastagens (Vitt \& Caldwell 2001). Gardner et al. (2007) encontraram maior diversidade de lagartos em áreas de floresta primária do que em áreas de floresta secundária e plantação de Eucalyptus spp. A redução de espécies em áreas de pastagens em Espigão do Oeste também foi observada para anfíbios anuros (Bernarde et al. 1999, Bernarde 2007) e para serpentes (Bernarde \& Abe 2006). Muitas espécies de lagartos não encontram condições de sobrevivência em ambientes onde a floresta primária foi retirada ou modificada (Heinen 1992, Vitt \& Caldwell 2001). Neste estudo foram registradas apenas oito espécies de lagartos nas áreas de pastagem. Dentre estas, Ameiva ameiva e 
Tabela 2. Número de indivíduos das espécies de lagartos capturados em armadilhas de interceptação e queda nos ambientes de floresta, floresta próximo de rio e em pastagem em Espigão do Oeste, Rondônia, Brasil.

Table 2. Individuals numbers of lizard species captured of lizards in pitfall traps with drift fences in forest, forest near a river and pasture environments at Espigão do Oeste, Rondônia, Brazil.

\begin{tabular}{|c|c|c|c|c|}
\hline \multirow[b]{2}{*}{ HOPLOCERCIDAE } & \multicolumn{4}{|c|}{$\begin{array}{c}\text { Floresta Floresta Pastagem Tota } \\
\text { Rio }\end{array}$} \\
\hline & & & & \\
\hline Enyalioides laticeps & 1 & - & - & 1 \\
\hline \multicolumn{5}{|l|}{ POLYCHROTIDAE } \\
\hline Anolis fuscoauratus & 3 & 4 & - & 7 \\
\hline A. nitens tandai & 1 & - & - & 1 \\
\hline A. punctatus & 3 & 4 & - & 7 \\
\hline A. transversalis & 1 & - & - & 1 \\
\hline \multicolumn{5}{|l|}{ LEIOSAURIDAE } \\
\hline Enyalius leechii & - & 1 & - & 1 \\
\hline \multicolumn{5}{|l|}{ TROPIDURIDAE } \\
\hline Plica plica & - & 3 & - & 3 \\
\hline Plica umbra & 2 & - & - & 2 \\
\hline Stenocercus roseiventris & 1 & 5 & - & 6 \\
\hline \multicolumn{5}{|l|}{ GEKKONIDAE } \\
\hline Coleodactylus amazonicus & 7 & 2 & - & 9 \\
\hline Gonatodes hasemani & 11 & 18 & - & 29 \\
\hline Gonatodes humeralis & 4 & 2 & 1 & 7 \\
\hline \multicolumn{5}{|l|}{ GYMNOPHTHALMIDAE } \\
\hline Cercosaura argulus & 6 & 2 & - & 8 \\
\hline Cercosaura eigenmanni & 7 & - & 1 & 8 \\
\hline Cercosaura ocellata & 11 & 8 & - & 19 \\
\hline Iphisa elegans elegans & 5 & 5 & - & 10 \\
\hline Leposoma osvaldoi & 4 & - & - & 4 \\
\hline \multicolumn{5}{|l|}{ TEIIDAE } \\
\hline Ameiva ameiva & 1 & - & 57 & 58 \\
\hline Kentropyx calcarata & - & 4 & - & 4 \\
\hline Kentropyx pelviceps & 33 & - & - & 33 \\
\hline Tupinambis teguixin & - & 1 & 1 & 2 \\
\hline \multicolumn{5}{|l|}{ SCINCIDAE } \\
\hline Mabuya nigropunctata & 7 & 3 & 7 & 17 \\
\hline Total de espécimes & 108 & 62 & 67 & 237 \\
\hline Total de espécies & 18 & 14 & 5 & 22 \\
\hline
\end{tabular}

Mabuya nigropunctata são espécies heliotérmicas e típicas de áreas abertas e Hemidactylus mabouia é uma espécie exótica e sinantrópica (Vanzolini 1986, Ávila-Pires 1995). As duas espécies de Gonatodes também foram registradas em ambientes abertos em Rondônia por Vanzolini (1986). Thecadactylus rapicauda e Cercosaura eigenmanni são espécies florestais que podem, eventualmente, ser encontradas em áreas abertas (Duellman 1987, Ávila-Pires 1995). Tupinambis teguixin é uma espécie que ocorre em florestas e em áreas abertas (Ávila-Pires 1995).

A ausência de espécies arborícolas (e.g., Anolis spp, Plica spp) e algumas terrícolas (e.g., Coleodactylus amazonicus, Cercosaurus argulus, C. eigenmanni) nas áreas de pastagem sugere que elas não consigam colonizar os ambientes abertos criados após o desmatamento. As razões sugeridas para isso são o aumento da taxa de predação, as dificuldades para a termorregulação, a perda de locais para abrigos e reprodução, a diminuição da oferta de alimento, o aumento de competição e a perda da serapilheira devido a redução da cobertura vegetal (Vitt et al. 1997, 1998, 2001, 2003a, b, c, 2005). Mesmo dentro das florestas, clareiras e trilhas abertas para a retirada de madeira favorecem a colonização por espécies de lagartos heliotérmicos que são potenciais competidoras e predadoras de algumas espécies de lagartos florestais (Vitt et al. 1998, Sartorius et al. 1999, Vitt \& Caldwell 2001).

Vinte e nove espécies de lagartos foram registradas para a área de estudo no município de Espigão do Oeste (RO), sendo a maioria encontrada em área de floresta. É provável que a conversão antrópica de florestas em áreas de pastagens impeça a colonização e a permanência de algumas espécies florestais terrícolas e arborícolas nesse ambiente. Assim, com a expansão da agropecuária no Estado de Rondônia e o conseqüente desmatamento, espécies de lagartos restritas a áreas florestais tenderão, provavelmente, a desaparecer localmente nessas áreas.

\section{Agradecimentos}

A Reginaldo A. Machado pela ajuda em algumas saídas de campo, sugestões e leitura do manuscrito. A Teresa Ávila-Pires pela confirmação e identificação de alguns espécimes. A Marcelo Kokubum pelo auxílio em algumas saídas de campo. Aos proprietários da Fazenda Jaburi Senhores Eduardo Garcia, Celso Garcia e família pelo apoio logístico. A Carlos Alberto Bernarde e família pelo apoio logístico em Espigão do Oeste. Ao Centro de Conservação e Manejo de Répteis e Anfíbios - RAN do IBAMA pelas licenças de coleta concedida (Processo 02001.006649/00-60; Licenças 246/2000-DIFAS/DIREC e 021/02-RAN). A CAPES pela bolsa de estudos concedida (PSB).

\section{Referências Bibliográficas}

AB' SABER, A.N. 1977. Os domínios morfoclimáticos da América do Sul. Boletim do Instituto de Geografia da Universidade de São Paulo 52:1-21.

ÁVILA-PIRES, T.C.S. 1995. Lizards of brazilian Amazonian (Reptilia: Squamata). Zool. Verh. 299:1-706.

ÁVILA-PIRES, T.C.S. \& VITT, L.J. 1998. A new species of Neusticurus (Reptilia: Gymnophthalmidae) from the Rio Juruá, Acre, Brazil. Herpetologica 54:235-245.

ÁVILA-PIRES, T.C.S. \& HOOGMOED, M.S. 2000. On two new species of Pseudogonatodes Ruthven, 1915 (Reptilia: Squamata: Gekkonidae), with remarks on the distribution of some other sphaerodactyl lizards. Zoologische Mededelingen 73:209-223.

ÁVILA-PIRES, T.C.S., HOOGMOED, M.S. \& VITT, L.J. 2007. Herpetofauna da Amazônia. In Herpetologia no Brasil II (L.B. Nascimento \& M.E. Oliveira, eds.). Sociedade Brasileira de Herpetologia, Belo Horizonte, p.13-43.

BERNARDE, P.S. 2007. Ambientes e temporada de vocalização da anurofauna no Município de Espigão do Oeste, Rondônia, Sudoeste da Amazônia - Brasil (Amphibia: Anura). Biota Neotrop. 7(2):87-92. http://www. biotaneotropica.org.br/v7n2/pt/fullpaper?bn01507022007+pt

BERNARDE, P.S. \& ABE, A.S. 2006. A snake community at Espigão do Oeste, Rondônia, Southwestern Amazon, Brazil. South American Journal of Herpetology 1(2):102-113.

BERNARDE, P.S., KOKUBUM, M.C.N., MACHADO, R.A. \& ANJOS, L. 1999. Uso de habitats naturais e antrópicos pelos anuros em uma localidade no Estado de Rondônia, Brasil (Amphibia: Anura). Acta Amazonica 29(4):555-562.

BRANDÃO, R.A. 2002. Avaliação ecológica rápida da herpetofauna nas Reservas extrativistas de Pedras Negras e Curralinho, Costa Marques, RO. Brasil Florestal 74:61-73.

CAMPBELL, H.W. \& CHRISTMAN, S.P. 1982. Field techniques for herpetofaunal community analysis, In Herpetological communities: a Symposium of the Society for the Study of Amphibians and Reptiles 
and the Herpetologists' League (N.J. Scott Jr., ed.). U. S. Fish Wildlife Service, Washington, p.193-200.

CECHIN, S.Z. \& MARTINS, M. 2000. Eficiência de armadilhas de queda (pitfall traps) em amostragem de anfíbios e répteis no Brasil. Revta bras. Zool. 17:729-740.

DUELLMAN, W.E. 1987. Lizards in an Amazonian rain forest community: resource utilization and abundance. National Geographic Research 3(4):489-500

DUELLMAN, W.E. 1990. Herpetofaunas in Neotropical rainforests: comparative composition, history, and resource use. In Four Neotropical Rainforests (A. H. Gentry, ed.). Yale University Press, New Haven, p.455-505.

GAINSBURY, A.M. \& COLLI, G.R. 2003. Lizard assemblages from natural cerrado enclaves in Southwestern Amazonia: the role of stochastic extinctions and isolation. Biotropica 35(4):503-519.

GARDNER, T.A., RIBEIRO-JÚNIOR, M.A., BARLOW, J., ÁVILA-PIRES, T.C.S., HOOGMOED, M.S. \& PERES, C.A. 2007. The value of primary, secondary, and plantation forests for a Neotropical herpetofauna. Conservation Biology 21(3):775-787.

HEINEN, J.T. 1992. Comparisons of the leaf litter herpetofauna in abandoned cacao plantations and primary rain forest in Costa Rica: some implications for faunal restoration. Biotropica 24(3):431-439.

MESQUITA, D.O., COSTA, G.C. \& COLLI, G.R. 2006. Ecology of an Amazonian savanna lizard assemblage in Monte Alegre, Pará state, Brazil. South American Journal of Herpetology 1(1):61-71.

NASCIMENTO, F.P., ÁVILA-PIRES, T.C.S.\& CUNHA, O.R. 1988. Répteis Squamata de Rondônia e Mato Grosso coletados através do programa Polonoroeste. Bol. Mus. Para. Emílio Goeldi, ser. Zool. 4:21-66.

NOGUEIRA, C. \& RODRIGUES, M.T. 2006. The genus Stenocercus (Squamata: Tropiduridae) in extra-amazonian Brazil, with the description of two new species. South American Journal of Herpetology 1(3):149-165.

OLIVEIRA, O.A. 2002. Geografia de Rondônia - espaço e produção. Dinâmica Ed. e Dist. Ltda, Porto Velho.

RODRIGUES, M.T. 2005. Conservação dos répteis brasileiros: os desafios para um país megadiverso. Megadiversidade 1(1):87-94.

RODRIGUES, M.T. \& AVILA-PIRES, T.C.S. 2005. New Lizard of the Genus Leposoma (Squamata, Gymnophthalmidae) from the Lower Rio Negro, Amazonas, Brazil. J. Herpetol. 49(4):541-546.

SANTOS-JR, A.P., FROTA, J.G. \& RIBEIRO, F.R.V. 2007. Reptilia, Squamata, Polychrotidae, Anolis nitens tandai: Distribution extension, new State record, and geographic distribution map. Check List 3(1):9-10.

SARTORIUS, S.S., VITT, L.J. \& COLLI, G.R. 1999. Use of natural and anthropogenically disturbed habitats in Amazonian rainforest by the teiid lizard Ameiva ameiva. Biological Conservation 90:91-101.

VANZOLINI, P.E. 1986. Levantamento herpetológico da área do Estado de Rondônia sob a influência da rodovia Br-364. Polonoreste/Ecologia Animal. Relatório de Pesquisa n ${ }^{\circ}, \mathrm{CNPq}$, Brasília.
VITT, L.J. \& CALDWELL, J.P. 1993. Ecological observations on cerrado lizards in Rondônia, Brazil. J. Herpetol. 27:46-52.

VITT, L.J. \& CALDWELL, J.P. 2001. The effects of logging on reptiles and amphibians of tropical forests. In The Cutting Edge: Conserving Wildlife in Logged Tropical Forests (R.A. Fimbel, A. Grajal \& J.G. Robinson, eds.). Columbia Univ. Press, New York, p.239-259.

VITT, L.J. \& COLLI, G.R. 1994. Geographical ecology of a Neotropical lizard: Ameiva ameiva (Teiidae) in Brazil. Can. J. Zool. 72:1986-2008.

VITT, L.J., ZANI, P.A. \& ÁVILA-PIRES, T.C.S. 1997. Ecology of the arboreal tropidurid lizard Tropidurus (= Plica) umbra in the Amazon region. Can. J. Zool. 75:1876-1882.

VITT, L.J., AVILA-PIRES, T.C.S., CALDWELL, J.P. \& OLIVEIRA, V.R.L. 1998. The impact of individual tree harvesting on thermal environments of lizards in amazonian rain forest. Conservation Biology 12:654-664.

VITT, L.J., SARTORIUS, S.S., ÁVILA-PIRES, T.C.S. \& ESPÓSITO, M.C. 2001. Life on the leaf litter: the ecology of Anolis nitens tandai in the Brazilian Amazon. Copeia 2001(2):401-412.

VITT, L.J., ÁVILA-PIRES, T.C.S., ESPÓSITO, M.C., SARTORIUS, S.S. \& ZANI, P.A. 2003a. Sharing amazonian rain-forest trees: ecology of Anolis punctatus and Anolis transversalis (Squamata: Polychrotidae). J. Herpetol. 37(2):276-285.

VITT, L.J., ÁVILA-PIRES, T.C.S., ZANI, P.A., SARTORIUS, S.S. \& ESPÓSITO, M.C. 2003b. Live above ground: ecology of Anolis fuscoauratus in the Amazon rain Forest, and comparisons with its nearest relatives. Can. J. Zool. 81:142-156

VITT, L.J., ÁVILA-PIRES, T.C.S., ZANI, P.A., ESPÓSITO, M.C. \& SARTORIUS, S.S. 2003c. Life at the interface: ecology of Prionodactylus oshaughnessyi in the Amazon and comparisons with P. argulus and $P$. eigenmanni. Can. J. Zool. 81:302-312.

VITT, L.J., SARTORIUS, S.S., ÁVILA-PIRES, T.C.S., \& ZANI, P.A. \& ESPÓSITO, M.C. 2005. Small in a big world: ecology of leaf-litter geckos in new world tropical forests. Herpetological Monographs 19:137-152.

VOGT, R.C., MOREIRA, G. \& DUARTE, A.C.O.C. 2001. Biodiversidade de répteis do bioma floresta Amazônica e Ações prioritarias para sua conservação. In: Biodiversidade na Amazônia Brasileira, Avaliação e ações prioritárias para a conservação, uso sustentavel e repartição de beneficios (J.P.R. Capobianco, Org.). Estação Liberdade: Instituto SocioAmbiental, São Paulo, p.89-96.

YUKI, R.N., GALATTI, U. \& ROCHA, R.A.T. 1999. Contribuição ao conhecimento da fauna de Squamata de Rondônia, Brasil, com dois novos registros. Bol. Mus. Para. Emílio Goeldi, ser. Zool.15:181-193.

Recebido em 24/06/07

Versão reformulada recebida em 06/12/07

Publicado em 10/01/08 


\section{Apêndice}

Tabela 1. Relação do material testemunho coletado na Fazenda Jaburi em Espigão do Oeste, Rondônia, Brasil, depositado na coleção Herpetológica do Museu Paraense Emílio Goeldi (MPEG) em Belém do Pará, Brasil.

Table 1. List of the voucher-specimens collected in the Jaburi Farm, Espigão do Oeste, Rondônia, Brazil, deposited in the Herpetological Collection of the Museu Paraense Emílio Goeldi (MPEG), Belém do Pará, Brazil.

\begin{tabular}{|c|c|}
\hline Espécie & Número de Coleção \\
\hline \multicolumn{2}{|l|}{ HOPLOCERCIDAE } \\
\hline Enyalioides laticeps & MPEG 21501 \\
\hline \multicolumn{2}{|l|}{ POLYCHROTIDAE } \\
\hline Anolis fuscoauratus & $\begin{array}{l}\text { MPEG 21474, MPEG 21491, MPEG 21900, MPEG 21901, } \\
\text { MPEG 21902, MPEG 21903, MPEG } 21904\end{array}$ \\
\hline Anolis nitens tandai & MPEG 21479, MPEG 21483, MPEG 21488, MPEG 21899 \\
\hline Anolis ortonii & MPEG 21490 \\
\hline Anolis punctatus & MPEG 21484, MPEG 21503, MPEG 21895, MPEG 21896, MPEG 21897 \\
\hline Anolis transversalis & MPEG 21475, MPEG 21905, MPEG 21906 \\
\hline \multicolumn{2}{|l|}{ LEIOSAURIDAE } \\
\hline Enyalius leechii & MPEG 21502, MPEG 21926, MPEG 21927 \\
\hline \multicolumn{2}{|l|}{ TROPIDURIDAE } \\
\hline Plica plica & MPEG 21493, MPEG 21939, MPEG 21940 \\
\hline Plica umbra & MPEG 21497, MPEG 21941 \\
\hline Stenocercus roseiventris & MPEG 21494, MPEG 21934, MPEG 21935, MPEG 21936 \\
\hline Stenocercus sineccasus & MPEG 21487 \\
\hline Uranoscodon superciliosus & MPEG 21949 \\
\hline \multicolumn{2}{|l|}{ GEKKONIDAE } \\
\hline Coleodactylus amazonicus & MPEG 21482, MPEG 21919 \\
\hline Gonatodes hasemani & $\begin{array}{l}\text { MPEG 21476, MPEG 21495, MPEG 21907, MPEG } 21908, \\
\text { MPEG 21909, MPEG 21910, MPEG 21911, MPEG } 21912\end{array}$ \\
\hline Gonatodes humeralis & $\begin{array}{l}\text { MPEG 21477, MPEG 21478, MPEG 21913, MPEG } 21914, \\
\text { MPEG 21915, MPEG } 21955\end{array}$ \\
\hline Thecadactylus rapicauda & MPEG 21954 \\
\hline \multicolumn{2}{|l|}{ GYMNOPHTHALMIDAE } \\
\hline Alopoglossus angulatus & MPEG 21951 \\
\hline Cercosaura argulus & MPEG 21498, MPEG 21920, MPEG 21921 \\
\hline Cercosaura eigenmanni & $\begin{array}{l}\text { MPEG 21473, MPEG 21481, MPEG 21499, } \\
\text { MPEG 21500, MPEG 21956, MPEG 21916, MPEG } 21918\end{array}$ \\
\hline Cercosaura ocellata bassleri & MPEG 21472, MPEG 21930, MPEG 21931, MPEG 21917 \\
\hline Iphisa elegans elegans & MPEG 21489, MPEG 21928, MPEG 21929 \\
\hline Leposoma osvaldoi & MPEG 21486, MPEG 21492, MPEG 21938 \\
\hline \multicolumn{2}{|l|}{ TEIIDAE } \\
\hline Ameiva ameiva & $\begin{array}{l}\text { MPEG 21942, MPEG 21944, MPEG 21944, MPEG } 21945, \\
\text { MPEG 21946, MPEG } 21947\end{array}$ \\
\hline Kentropyx calcarata & MPEG 21485, MPEG 21923, MPEG 21924, MPEG 21925 \\
\hline Kentropyx pelviceps & MPEG 21480, MPEG 21922, MPEG 21922 \\
\hline Tupinambis teguixin & MPEG 21932, MPEG 21933 \\
\hline \multicolumn{2}{|l|}{ Scincidae } \\
\hline Mabuya nigropunctata & MPEG 21496, MPEG 21952, MPEG 21953 \\
\hline
\end{tabular}

